\title{
Spatially resolved correlation between glass structure and refractive index modifications resulting from irradiation of chalcogenide glass by femtosecond pulse train
}

\author{
P. Masselin ${ }^{1}$, D. Le Coq $^{2}$, A. Cuisset ${ }^{1}$ and E. Bychkov ${ }^{1}$ \\ ${ }^{1}$ Lab. Physico-Chimie de l'Atmosphère, Université du Littoral Côte d'Opale \\ 189A Av Maurice Schumann, 59140 Dunkerque France \\ ${ }^{2}$ Institut des Sciences Chimiques de Rennes - Unité Verres et Céramiques CNRS UMR 6226 \\ Campus de Beaulieu - Bat 10B - 35042 Rennes Cedex \\ masselin@univ-littoral.fr
}

\begin{abstract}
A detailed analysis of 2D $\mu$-Raman mapping of refractive index variation $(\Delta n)$ induced by femtosecond filament in the bulk of a ternary (Ge-Ga-S) chalcogenide glass is presented. The results demonstrate strong structural modifications. $\mathrm{S}_{3} \mathrm{Ga}-\mathrm{GaS}_{3}$ ethane like units are transformed into triclusters of $\mathrm{Ga} / \mathrm{S}$. Also the decrease of the population of $\mathrm{GeS}_{4 / 2}$ tetrahedrons implies the formation of mixed triclusters $\mathrm{T} / \mathrm{S}$ where $\mathrm{T}=\mathrm{Ga}$ and/or Ge. Finally, the refractive index variation is correlated to the structural modifications.
\end{abstract}

\section{Introduction}

In chalcogenide glass, femtosecond pulse can easily form a filament because of the high optical nonlinearities of the glass. From the resulting light-matter interaction, a refractive variation is produced with a complex morphology alternating positive and negative sign. In order to understand this behaviour, it is important to study the structural modifications that lead to the refractive index variation.

Therefore we perform a 2D $\mu$-Raman analysis of $\Delta n$. Four structural units are identified to contribute to the Raman spectra. By following the spatial evolution of the four contributions, a correlation between $\Delta n$ and the contribution of each group to $\Delta n$ is obtained.

\section{Experimental procedure}

The glass composition is $80 \mathrm{GeS}_{2}-20 \mathrm{Ga}_{2} \mathrm{~S}_{3}$ and it has been synthesized according to classical melt and quenching technique [1]. The sample is irradiated by a high repetition rate femtosecond Ti:Sa laser. The pulse energy is moderate $(2 \mathrm{~nJ})$ and the pulse duration is measured to be around $\sim 200 \mathrm{fs}$. The beam is focused inside the bulk of the sample by a $\mathrm{X} 5$ microscope objective and the exposure duration is set to 1 second without translation of the sample. A photography of $\Delta n$ is shown in figure 1 along with its quantitative reconstruction according the procedure described in [2].
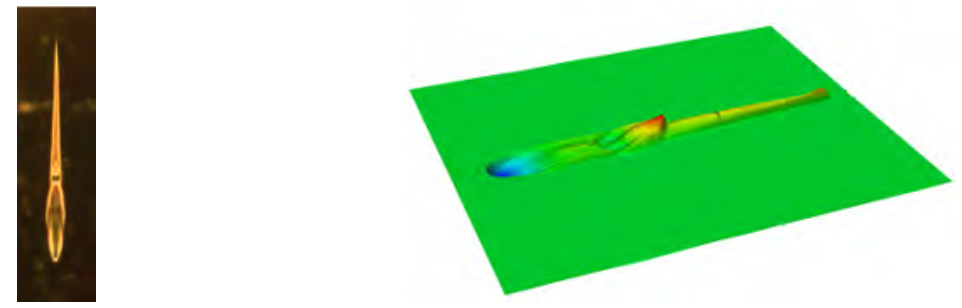

Fig. 1: Photography and reconstruction of $\Delta n$ induced y femtosecond pulse train in the bulk of $80 \mathrm{GeS}_{2}-20 \mathrm{Ga}_{2} \mathrm{~S}_{3}$ glass.

After irradiation, the sample is cleaved to bring $\Delta n$ on the surface. Raman spectra are recorded using a Jobin Yvon Raman micro-spectrometer. A mapping of $326 \times 68 \mu \mathrm{m}^{2}$ surface is obtained by translating the sample with a 
resolution of $1 \mu \mathrm{m}$ in the direction perpendicular to the writing laser beam, and $2 \mu \mathrm{m}$ in the parallel direction. The total number of Raman spectra is more than 11,000.

On the basis of DFT calculation we determine the structural unit that contribute to the Raman spectra. We identify the presence of corner sharing and edge sharing tetrahedron of $\mathrm{GeS}_{4}, \mathrm{~S}_{3} \mathrm{Ga}-\mathrm{GaS}_{3}$ ethane like unit and tricluster of $\mathrm{Ga} / \mathrm{S}$. Every group has a well resolved vibration mode from which the behaviour of its contribution can unambiguously be determined. All the Raman spectra are fitted considering only four parameters corresponding to the amplitude of each structural unit contribution.

\section{Results}

In fig. 2, the amplitude of the contribution of the four structural units are shown. It is clearly seen that the laser irradiation induces a decrease of the population of $\mathrm{GeS}_{4}$ tetrahedrons and a large increase of $\left[\mathrm{TS}_{1 / 3} \mathrm{~S}_{3 / p}\right]_{3}$ triclusters.

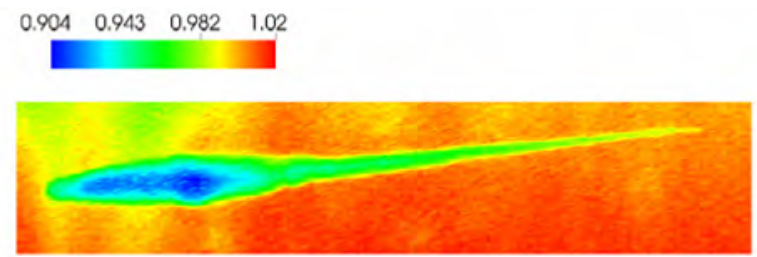

(a) $\mathrm{CS}_{-} \mathrm{GeS}_{4}$ tetrahedron sharing corner

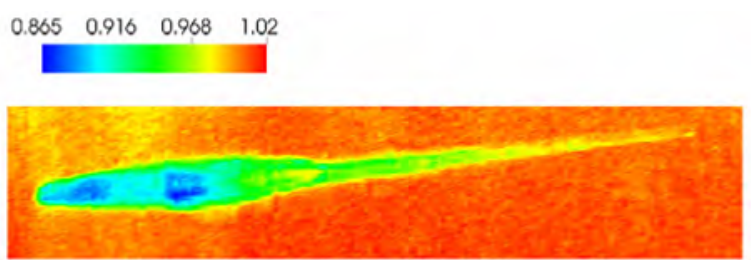

(c) $\mathrm{S}_{3} \mathrm{Ga}-\mathrm{GaS}_{3}$ ethane like group

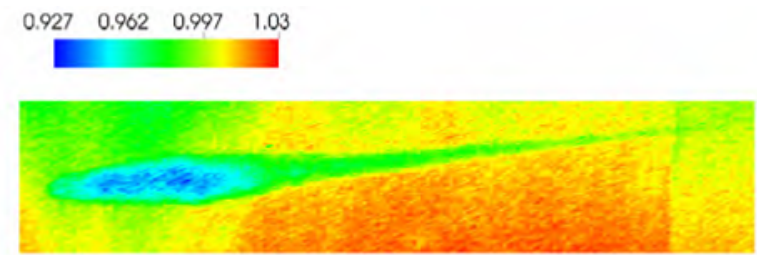

(b) $\mathrm{ES}_{-}-\mathrm{GeS}_{4}$ tetrahedron sharing edge.

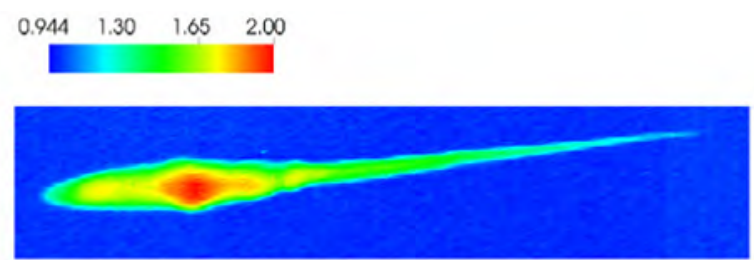

(d) $\left[\mathrm{GaS}_{1 / 3} \mathrm{~S}_{3 / p}\right]_{3}$ triclusters, where $p=2$ or 3 .

Fig. 2: Mapping of the relative contribution of the four groups into the Raman spectra.

By comparing the $\Delta n$ (fig. 1) and the Raman mapping figures, it is clear that the formation of a positive $\Delta n$ is associated with the formation of triclusters. We attribute the negative $\Delta n$ to the formation of nanovoids resulting from Coulombian micro-explosion.

\section{Conclusion}

In conclusion we perform a 2D $\mu$-Raman mapping of laser irradiation of chalcogenide glass. Light matter interaction results in strong chemical bonds reordering from $\mathrm{S}_{3} \mathrm{Ga}-\mathrm{GaS}_{3}$ ethane like units into $\left[\mathrm{GaS}_{1 / 3} \mathrm{~S}_{3 / p}\right]_{3}$ triclusters.

The tail of positive $\Delta n$ is shown to be due to the higher efficiency of the tricluster tetrahedral packing leading to a higher density. As the number of free carriers generated in the filament increases, Coulombian micro-explosions occur and induce the formation of nanovoids responsible of the $\Delta n$ sign reversal.

\section{References}

[1] P. Masselin, D. Le Coq, L. Calvez, E. Petracovschi, E. Lépine, E. Bychkov, and X. Zhang, "CsCl effect on the optical properties of the $80 \mathrm{GeS}_{2}-20 \mathrm{Ga}_{2} \mathrm{~S}_{3}$ base glass," Applied Physics A: Materials Science \& Processing 106, 697-702 (2012). 10.1007/s00339-011-6668-6.

[2] E. Ampem-Lassen, S. T. Huntington, N. M. Dragomir, K. A. Nugent, and A. Roberts, "Refractive index profiling of axially symmetric optical fibers: a new technique," Opt. Express 13, 3277-3282 (2005). 